\title{
The endonasal patient reference tracker: a novel solution for accurate noninvasive electromagnetic neuronavigation
}

\author{
Ayguel Wurzer, MD,, Georgi Minchev, MD, ${ }^{1}$ Claudia Cervera-Martinez, MD, ${ }^{1}$ \\ Alexander Micko, MD, PhD, ${ }^{1}$ Gernot Kronreif, $\mathrm{PhD},{ }^{2}$ and Stefan Wolfsberger, MD${ }^{1}$ \\ 1Department of Neurosurgery, Medical University Vienna; and ²Austrian Center of Medical Innovation and Technology, Wiener \\ Neustadt, Austria
}

\begin{abstract}
OBJECTIVE Electromagnetic (EM) navigation provides the advantages of continuous guidance and tip-tracking of instruments. The current solutions for patient reference trackers are suboptimal, as they are either invasively screwed to the bone or less accurate if attached to the skin. The authors present a novel EM reference method with the tracker rigidly but not invasively positioned inside the nasal cavity.

METHODS The nasal tracker (NT) consists of the EM coil array of the AxiEM tracker plugged into a nasal tamponade, which is then inserted into the inferior nasal meatus. Initially, a proof-of-concept study was performed on two cadaveric skull bases. The stability of the NT was assessed in simulated surgical situations, for example, prone, supine, and lateral patient positioning and skin traction. A deviation $\leq 2 \mathrm{~mm}$ was judged sufficiently accurate for clinical trial. Thus, a feasibility study was performed in the clinical setting. Positional changes of the NT and a standard skin-adhesive tracker (ST) relative to a ground-truth reference tracker were recorded throughout routine surgical procedures. The accuracy of the NT and ST was compared at different stages of surgery.
\end{abstract}

RESULTS Ex vivo, the NT proved to be highly stable in all simulated surgical situations (median deviation $0.4 \mathrm{~mm}$, range $0.0-2.0 \mathrm{~mm}$ ). In 13 routine clinical cases, the NT was significantly more stable than the ST (median deviation at procedure end $1.3 \mathrm{~mm}$, range $0.5-3.0 \mathrm{~mm}$ vs $4.0 \mathrm{~mm}$, range $1.2-11.2 \mathrm{~mm}, p=0.002$ ). The loss of accuracy of the ST was highest during draping and flap fixation.

CONCLUSIONS Application of the EM endonasal patient tracker was found to be feasible with high procedural stability ex vivo as well as in the clinical setting. This innovation combines the advantages of high precision and noninvasiveness and may, in the future, enhance EM navigation for neurosurgery.

https://thejns.org/doi/abs/10.3171/2020.4.JNS20394

KEYWORDS nasal tracker; electromagnetic navigation; dynamic reference frame; surgical technique

$\mathrm{B}$ ECAUSE of their ability to provide intraoperative image guidance with high accuracy, frameless navigation systems have become an indispensable tool for cranial neurosurgical procedures. ${ }^{1}$ As in automotive navigation, modern surgical navigation guidance should be accurate and continuously available. It should also be noninvasive, that is, without the need for a rigid head clamp or invasive patient tracker fixation. During procedures, it should provide continuous feedback about the actual instrument tip position.

Optical tracking of infrared light-reflecting spheres combines cost-effectiveness with high usability and is therefore the most widely used navigation technology in neurosurgery today. However, optical tracking is not without limitations. The requirement for a constant visible connection among the patient reference tracker, the tracked instrument, and the camera bar renders continuous instrument tracking difficult. ${ }^{2}$

Electromagnetic (EM) tracking detects the electric current induced in a 3D coil array brought inside an EM field to calculate the instrument's position. If ferromagnetic interference is minimized, this technique has no interruptions in the line of sight, can be completely hidden under the draping, and thus can be used for constant guidance and tip-tracking of instruments..$^{3-5}$

The function of a patient reference tracker, also termed

ABBREVIATIONS DRF = dynamic reference frame; EM = electromagnetic; NT = nasal tracker (endonasal patient reference tracker); RT = reference tracker (for ground truth); $\mathrm{ST}=$ skin-adhesive tracker.

SUBMITTED February 10, 2020. ACCEPTED April 21, 2020.

INCLUDE WHEN CITING Published online July 17, 2020; DOI: 10.3171/2020.4.JNS20394. 
"dynamic reference frame" (DRF), is to define the exact position of the patient's head in space. Therefore, it requires a stable connection with the patient's head. If the DRF's position relative to the patient's head changes during a neurosurgical procedure, navigation becomes inaccurate. In optic navigation, the DRF is rigidly fixed to the patient's head mostly via the skull clamp. Thus, invasive three-pin head fixation is usually required. Registration can be performed only after patient positioning, andespecially in the prone position-may represent a cumbersome task with limited accuracy. Further, navigationguided positioning is not possible, nor is repositioning of the patient during the procedure. If head movement occurs inadvertently (e.g., due to force applied during the procedure), re-registration with specially defined points is needed or navigation is lost.

In EM navigation, the DRF is usually directly fixed to the patient's head, obviating the need for rigid head fixation. ${ }^{3}$ Consequently, the patient's head can be repositioned at any time during surgery without the need for re-registration. The currently available DRFs for EM navigation are fixed to the patient's head via bone screws or skin adhesive. Bone-mounted trackers have higher registration accuracy but require a skin incision and are therefore unacceptably invasive for routine use. Skin-adhesive trackers (STs) are noninvasive but have lower accuracy due to skin mobility. ${ }^{6}$ Consequently, the existing DRF solutions for EM navigation are suboptimal and limit wider use of this navigation technology. Therefore, alternative noninvasive and stable fixation methods for the EM DRF are needed.

Here, we present a novel endonasal DRF that combines the noninvasiveness of a nasal tamponade with the stability provided by the osseous nasal structures. The aim of this study was to test this endonasal patient reference tracker ex vivo and to assess the feasibility of its application and stability in the routine clinical setting.

\section{Methods}

\section{Description of the Nasal Tracker}

The EM tracking device used in this study is a waterproof, 5-mm-diameter, 2-cm-long, rod-type, 3D coil array for position detection within the field created by the EM emitter. It is contained in various instruments and trackers of the AxiEM navigation system (Medtronic USA). This device was manually removed from the housing of the wing-type ST and plugged into the conduit of a standard nasal dressing (Merocel, Medtronic USA) after removal of its silicone airway tube until close to the distal end (Fig. 1). With the aid of a short nasal speculum, this "nasal tracker" (NT) is inserted through the wider of the two nostrils into the posterior osseous part of the inferior nasal meatus. The cable is secured to the philtrum via a strain relief loop and surgical tape to prevent inadvertent malpositioning or removal by drag on its cable during a procedure (Fig. 2).

\section{Preclinical Cadaver Study}

Prior to clinical application, a proof-of-concept study was performed ex vivo in the four nostrils of two cadaveric skull bases to test the applicability and stability of the NT assembly inside the nasal cavity. We tested its position deviation from a ground-truth reference tracker (RT). An invasive EM tracker was used as the RT and was fixed to the cadaveric frontal skull base via two bone screws. The NT was inserted as described above. Position values of the two trackers were obtained from the navigation system's interface via ethernet.

The distance between the two trackers was initially set to 0 and was continuously assessed and recorded by custom-designed software throughout the experiment. Instability of the NT-resulting in relative movements between the trackers-was defined by deviation from the baseline 0 . The stability of the NT was tested in various simulated surgical situations such as head rotation (for prone, supine, and lateral patient positioning), head flexion, and draping $(0.5-\mathrm{kg}$ weight drag from lateral and top, and manual nasal deformation). A deviation $\leq 2 \mathrm{~mm}$ was judged sufficiently accurate to proceed to a clinical trial.

\section{Clinical Feasibility Study}

To assess the feasibility of the NT in the clinical setting and compare its stability to that of the existing ST, the NT was applied in routine adult neurosurgical cases. Only those cases without a history of complex nasal or oral surgery or extensive nasal or oral injuries were included. As only ferromagnetic skull clamps were available during the study period, we only included those procedures with the patient placed prone to minimize interference effects.

All patients gave informed consent to participate in the study, which had been approved by the Ethics Committee of the Medical University of Vienna and the Austrian Agency for Health and Food Safety.

\section{Patient Setup}

An RT rigidly attached to the patient's head served as ground truth for surgical navigation (Fig. 3A). The RT was either fixed to the head clamp or directly mounted to the skull via invasive bone screws. The latter, more invasive setup requiring a skin incision was used if spring retractors were applied to the skin flap, which can exert torque on the head clamp and render measurements inaccurate (see Stieglitz et al. ${ }^{7}$ ), or in cases of nonrigid head positioning without a clamp.

The NT was fixed as described above. Data about the feasibility of the assembly, endonasal positioning, and fixation of the NT were collected. The ST was attached to the patient's skin at a position judged most unaffected by surgery within the EM detection field.

Position values of the three trackers were obtained from the navigation system's StealthLink (Medtronic) interface via ethernet and concurrently recorded using custom-designed personal computer-based software (Austrian Center of Medical Innovation and Technology). To assess the stability of the NT, this software continuously recorded the NT's position relative to the RT. To compare the NT's stability to that of the ST, we continuously recorded the position of the ST relative to the RT in parallel. Initially set to 0 , the software visualized any deviation of the NT and ST relative to the RT in a time-dependent graph (Fig. 3B). Measurements were recorded at $5 \mathrm{~Hz}$ until the beginning of the microsurgery and $0.5 \mathrm{~Hz}$ until the end of 

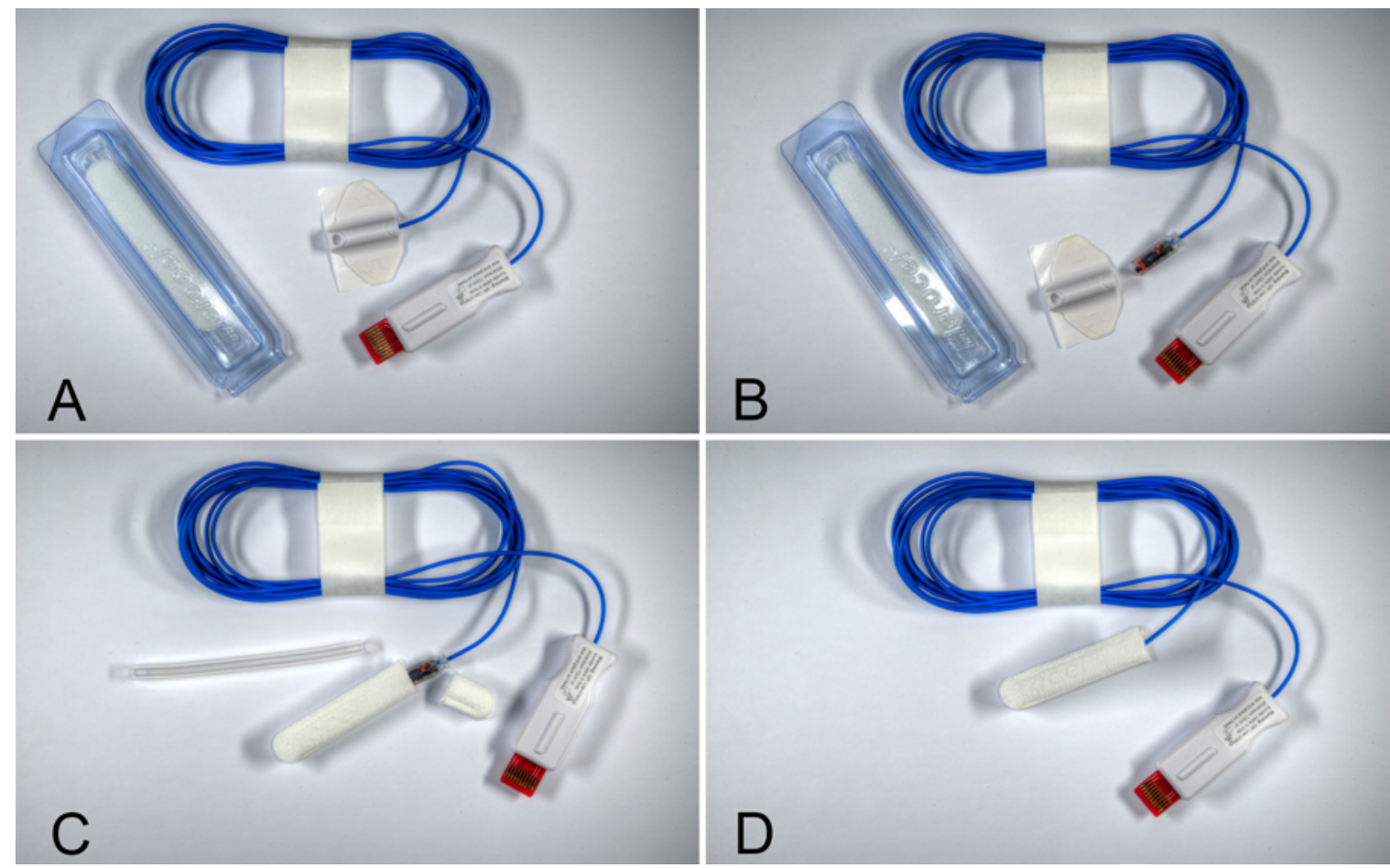

FIG. 1. NT assembly. The EM tracking array was manually removed from the housing of the wing-type ST (A and B) and plugged close to the distal end of a standard nasal dressing after removal of its silicone airway tube ( $C$ and $D)$.

the microsurgical part of the procedure (i.e., removal of the operating microscope). To correlate the measurements with surgical tasks, labels were manually entered at the following prespecified events: sterile preparation, sterile draping, start of procedure, skin incision, flap fixation, craniotomy, beginning of microsurgery, and end of microsurgery. At the end of the procedure, any complications in removal of the NT were noted. At follow-up, nasal comfort was assessed via patient interviews.

\section{Statistical Analysis}

For statistical analysis, SPSS software (version 26.0, IBM Corp.) was used. Values are given as the median and range. For the clinical trial, we compared the stability of the NT and the ST using the paired t-test. A p value $<0.05$ was considered statistically significant.

\section{Results}

\section{Assembly and Application of the NT}

Manual assembly of the NT was feasible in all cases; the EM tracker was found to fit perfectly inside the nasal tamponade without clearance. For atraumatic endonasal insertion, we applied antibiotic ointment on the surface of the nasal tamponade-immersion in water was found to be less useful due to a tendency of the soaked tamponade to deform on insertion.

\section{Preclinical Cadaver Study}

Application of the NT was feasible in all four nostrils of both cadaveric heads. Overall, the NT proved highly stable in all simulated surgical situations (median deviation $0.4 \mathrm{~mm}$, range $0.0-2.0 \mathrm{~mm}$ ). The greatest deviation was observed during the simulation of prone, supine, and lateral positioning by head rotation (median deviation 1.4 $\mathrm{mm}$, range $0.3-2.0 \mathrm{~mm}$ ). Ante- and retroflexion and draping with a one-sided $0.5-\mathrm{kg}$ weight attachment resulted in only submillimetric deviations (Table 1).

\section{Clinical Feasibility Study}

The NT was tested in 13 routine clinical cases. For patient and procedure characteristics see Table 2. After inducing general anesthesia and patient positioning, we first attached the RT to the head clamp in cases with skin retractors $(n=6)$ or directly to the bony skull in cases of spring-hook flap fixation or if no head clamp was used $(\mathrm{n}=$ 7). Then, patient-to-image registration was performed. After the collection of sufficient surface points, a landmark check was performed on the glabella, lateral canthi, and external auditory canals. If judged inaccurate, registration was repeated. After palpation of the inferior nasal meatus, the NT was always inserted into the larger nasal cavity. Application of the NT was feasible in all 13 cases. The ST was attached to the patient's skin at a bald spot most unaffected by the surgery (i.e., contralateral to the skin incision). At the start of the procedure, the software was started and the distances between the RT and test trackers were automatically set to 0 .

Overall, the NT was significantly more stable than the ST (median deviation at the end of microsurgery: $1.3 \mathrm{~mm}$, range $0.5-3.0 \mathrm{~mm}$ vs $4.0 \mathrm{~mm}$, range $1.2-11.2 \mathrm{~mm}, \mathrm{p}=$ 0.002). The gain of deviation was highest for the ST dur- 

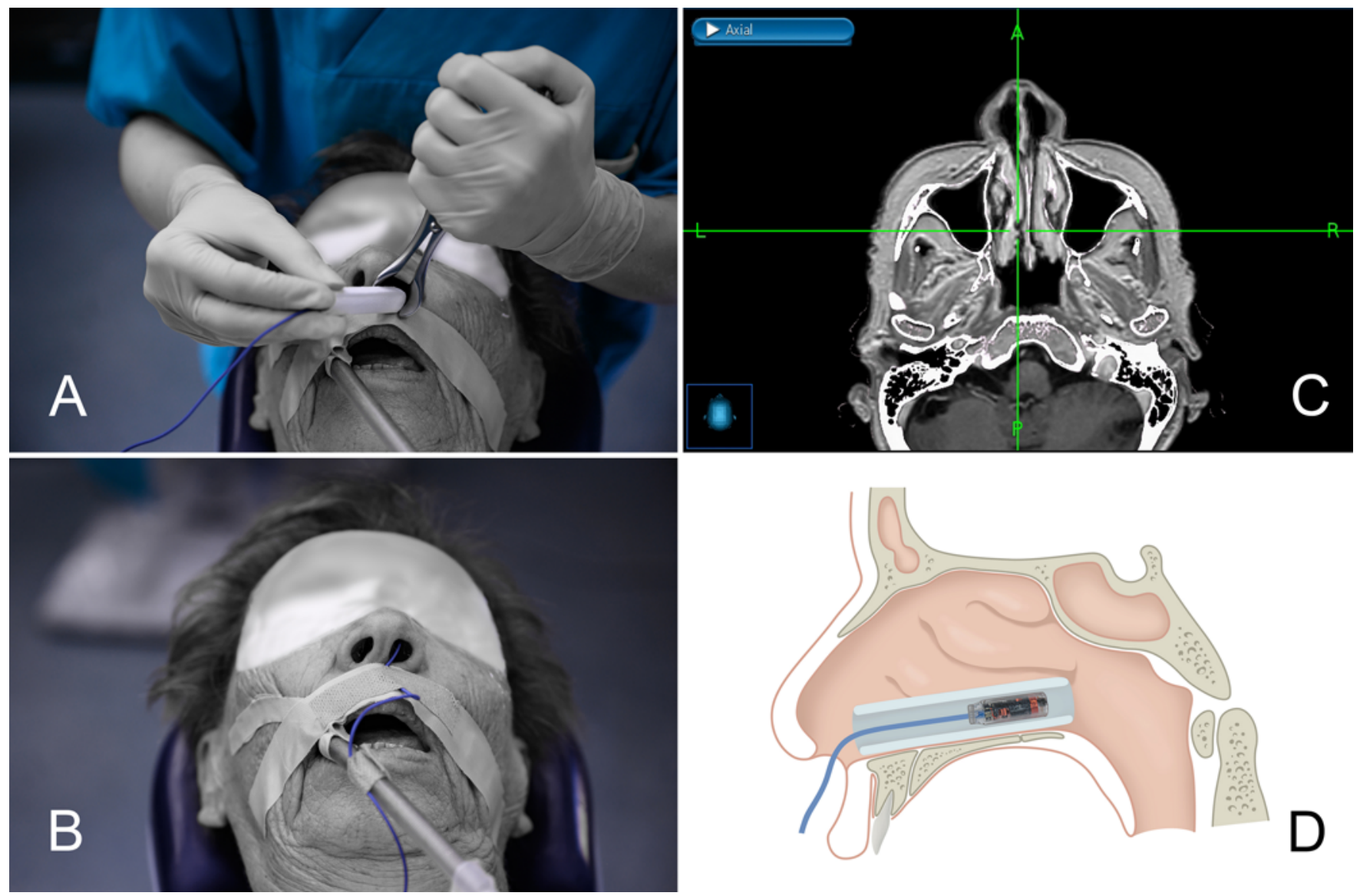

FIG. 2. Application of the NT. Using a nasal speculum, the NT was inserted into the wider of the two nostrils (A), and the cable was secured to the philtrum via a strain relief loop to prevent inadvertent malpositioning by drag on its cable (B). The final position of the NT in the posterior part of the inferior nasal meatus is shown by direct navigation of the tracker (C) and in a drawing (D). Image in panel D copyright Stefan Wolfsberger. Published with permission.
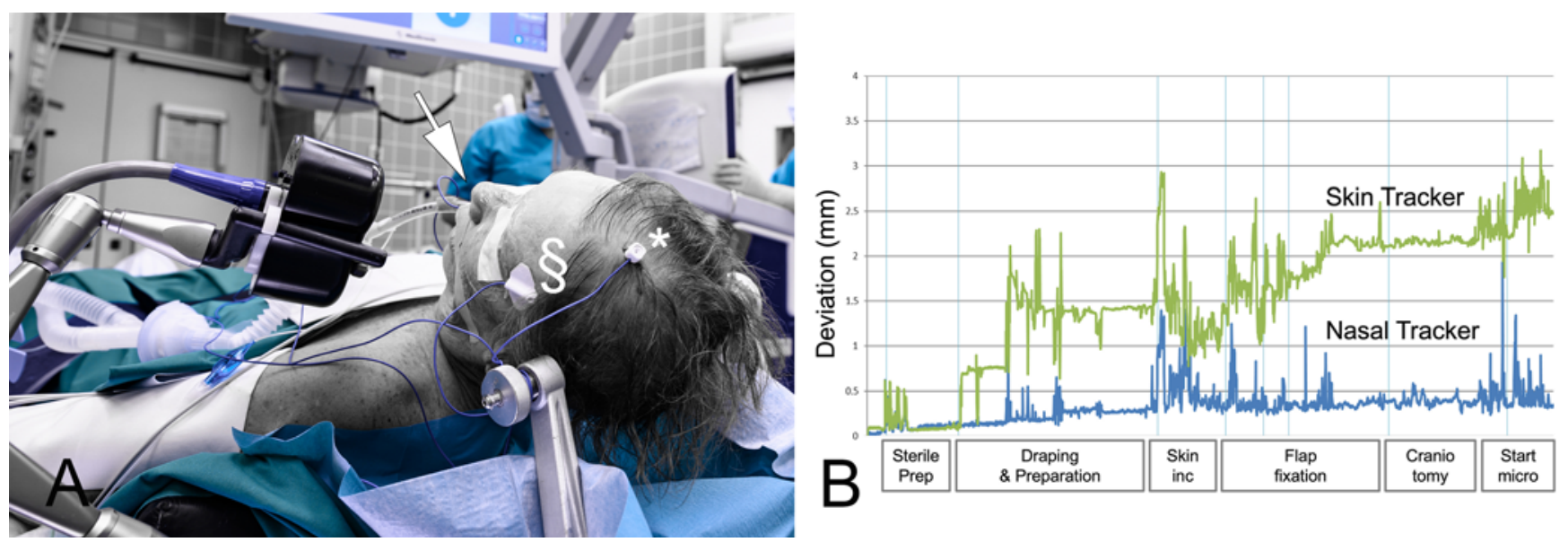

FIG. 3. A: Clinical feasibility study setup. An RT $\left(^{*}\right)$ was rigidly attached to the patient's head and served as ground truth for surgical navigation. The ST (§) was glued to the patient's skin at a bald spot judged most unaffected by surgery within the EM detection field. The NT (arrow) was inserted into the larger nasal cavity. B: Output of the recording software. The deviation of the ST and NT relative to the reference was recorded throughout the procedure and labeled at predefined surgical tasks. inc $=$ incision; micro = microsurgery. 
TABLE 1. Results of the proof-of-concept cadaveric study

\begin{tabular}{|c|c|c|}
\hline \multirow[b]{2}{*}{ Task } & \multicolumn{2}{|c|}{ Deviation $(\mathrm{mm})$} \\
\hline & Median & Range \\
\hline \multicolumn{3}{|c|}{ Drape w/ load $(0.5 \mathrm{~kg})$} \\
\hline Rt & 0.0 & $0.0-0.1$ \\
\hline $\mathrm{Lt}$ & 0.1 & $0.0-0.1$ \\
\hline Top & 0.1 & $0.0-0.2$ \\
\hline \multicolumn{3}{|c|}{ Manually applied force } \\
\hline To rt & 0.4 & $0.1-0.6$ \\
\hline To lt & 1.5 & $0.6-1.6$ \\
\hline \multicolumn{3}{|l|}{ Head rotation } \\
\hline $90^{\circ}$ & 0.9 & $0.3-1.6$ \\
\hline $180^{\circ}$ & 1.3 & $0.3-1.8$ \\
\hline $270^{\circ}$ & 1.5 & $0.5-1.5$ \\
\hline \multicolumn{3}{|l|}{ Head flexion } \\
\hline Anteflexion & 0.4 & $0.0-0.5$ \\
\hline Retroflexion & 0.5 & $0.2-1.0$ \\
\hline
\end{tabular}

ing draping (median deviation gain $1.3 \mathrm{~mm}$, range $0.1-3.5$ $\mathrm{mm}$ ) and flap fixation (median deviation gain $1.6 \mathrm{~mm}$, range $0.0-7.4 \mathrm{~mm}$ ), but not for the NT (median deviation gain $0.3 \mathrm{~mm}$, range $0.0-1.3 \mathrm{~mm}$ and $0.2 \mathrm{~mm}$, range $0.0-$ $2.8 \mathrm{~mm})$.

After craniotomy, at the start of the microsurgical procedure, the absolute deviation from baseline was significantly lower for the NT than for the ST (median $1.0 \mathrm{~mm}$, range $0.3-4.0 \mathrm{~mm}$ vs $4.3 \mathrm{~mm}$, range $1.3-14.4 \mathrm{~mm}, \mathrm{p}=$ $0.002)$. This deviation remained more stable throughout the microsurgical procedure for the NT than for the ST (median deviation gain $0.3 \mathrm{~mm}$, range $0.0-1.0 \mathrm{~mm}$ vs 0.3 $\mathrm{mm}$, range $0.0-11.2 \mathrm{~mm}$ ); however, the difference between the two was not statistically significant.

For absolute deviation of the test tracker position from baseline at the labeled events see Fig. 4, and for the relative deviation change between events see Table 3 .

\section{Complications}

Epistaxis at insertion of the NT was never observed. On removal of the NT, we encountered one case of minor epistaxis that resolved spontaneously without intervention. At follow-up, nasal comfort was maintained according to patient interviews.

\section{Discussion}

\section{Background Summary}

EM navigation technology provides the possibility for continuous instrument tip-tracking without rigid head fixation and is therefore preferred in otolaryngological and craniofacial surgery. ${ }^{8,9}$ To overcome the line-of-sight issue of optic tracking and allow continuous instrument guidance, we have previously suggested EM navigation technology for neurosurgical procedures as well. ${ }^{4}$ Current EM navigation systems have been reported to be as accurate as optic tracking in the laborator $y^{10}$ as well as in the clinical setting. ${ }^{11}$ They may even provide submillimetric accuracy $^{9,12}$ if registration error is minimized by bone-screw
TABLE 2. Clinical characteristics of 13 patients who underwent EM navigation

\begin{tabular}{lc}
\hline \multicolumn{1}{c}{ Characteristic } & Value \\
\hline Median age in yrs (range) & $55(37-79)$ \\
\hline M/F ratio & $1.0: 2.3$ \\
\hline Diagnosis & $7(54 \%)$ \\
\hline Meningioma & $5(38 \%)$ \\
\hline Glioma & $1(8 \%)$ \\
\hline Pituitary adenoma & $13(100 \%)$ \\
\hline Transcranial resection & $13(100 \%)$ \\
\hline Patient position supine & \\
\hline Head rotatation & $3(23 \%)$ \\
\hline $0^{\circ}$ & $5(38 \%)$ \\
\hline $1^{\circ}-45^{\circ}$ & $5(38 \%)$ \\
\hline $46^{\circ}-90^{\circ}$ & \\
\hline Head fixation & $12(92 \%)$ \\
\hline Head clamp & $1(8 \%)$ \\
\hline None (horseshoe headrest) & $7(54 \%)$ \\
\hline RT & $6(46 \%)$ \\
\hline Invasive (bone anchored) & $3.5(2.0-5.5)$ \\
\hline Noninvasive (skull clamp)
\end{tabular}

registration and a stable emitter/DRF. Clinically available DRFs are fixed to the patient via bone screws or skin adhesive. Bone-mounted trackers have higher registration accuracy but require a skin incision that is unacceptably invasive for routine use. STs are noninvasive but prone to lower accuracy due to skin mobility. ${ }^{6}$

We present a novel endonasal DRF that combines the noninvasiveness of a nasal tamponade with the stability of the osseous nasal structures. According to data in the present study, application of the NT is feasible and noninvasive and has continuous high stability in the routine clinical setting.

\section{Electromagnetic Patient Tracker Solutions}

There have been previous attempts to increase accuracy through the development of stable DRF fixations. The DigiPointeur (Collin Medical) is an EM navigation system for ear, nose, and throat (ENT) surgery. Its oral device combining emitter and DRF (Buccostat) was reported to drift in up to $20 \%$ of procedures. ${ }^{13}$ Bernardeschi et al. developed an alternative emitter-DRF that was implanted via a $1-\mathrm{cm}$ skin incision and two 4-mm-diameter titanium screws close to the retroauricular surgical area..$^{14}$ Use of this bone screw-based registration resulted in significant improvement $(<1 \mathrm{~mm})$ in cases of lateral skull base surgery. Data about continuous accuracy were not reported.

For the highest accuracy, as required in temporal bone surgery, a bone-fixed DRF seems warranted. In the majority of neurosurgical cases, however, this setup seems too invasive. A deviation $\leq 2 \mathrm{~mm}$ throughout a procedure, as in our current study, is low compared to that with optical navigation in the clinical setting. ${ }^{7}$ We consider the accuracy of the NT appropriate for routine neurosurgical procedures. 


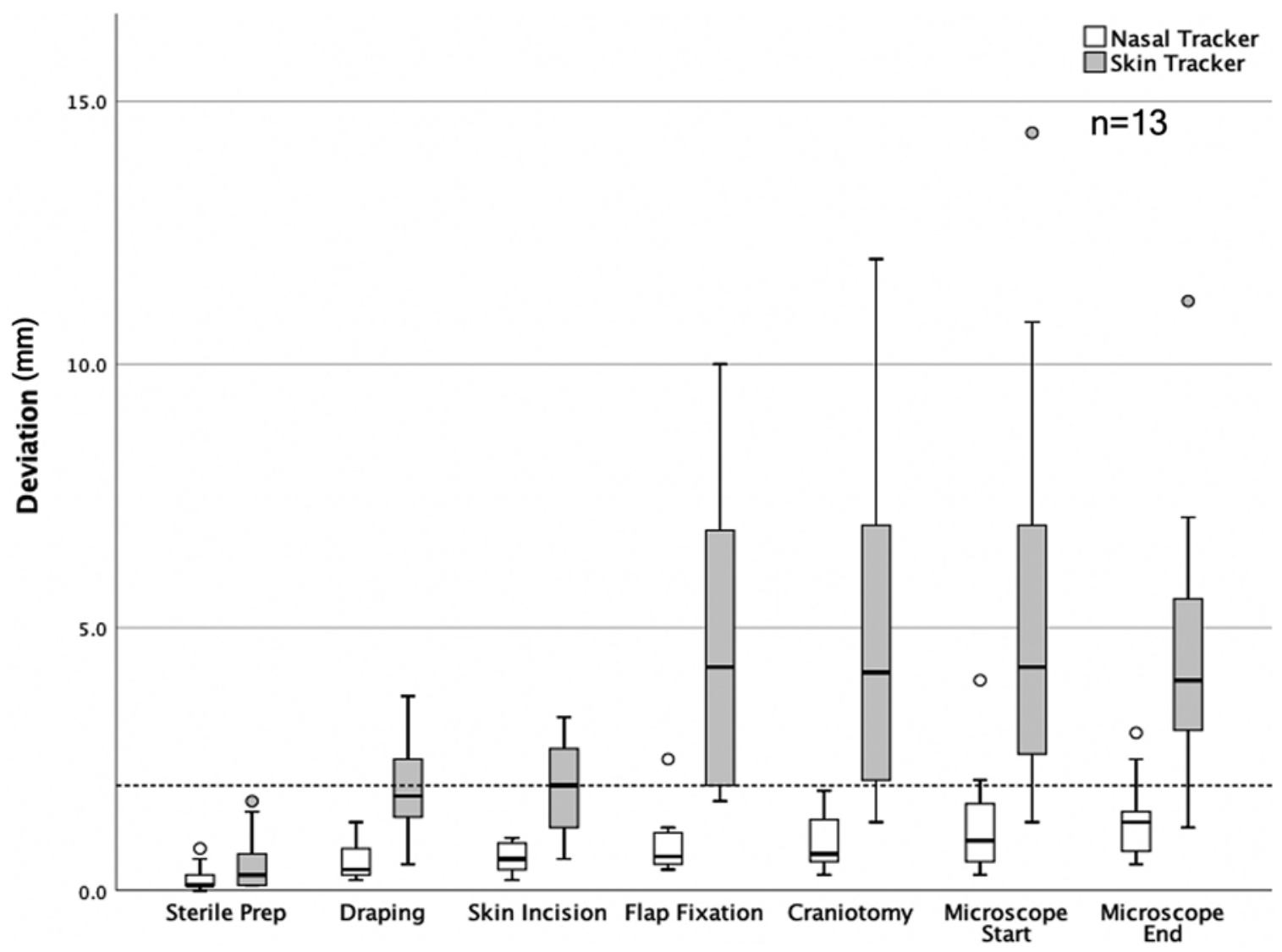

FIG. 4. Absolute deviation of the NT and ST positions from baseline at predefined time points. The greatest gain was during flap fixation, followed by draping for the ST, but not for the NT. Note that the median deviation of the NT never exceeded $2 \mathrm{~mm}$ (dotted line), whereas the ST exceeded $4 \mathrm{~mm}$ after flap fixation. $\mathrm{n}=$ number of cases.

Kral et al. developed an EM tracker fixed at the posterior edge of the vomer..$^{15}$ This DRF was equipped with 6 hooks for fixation in the nasal mucosa. Compared to a skin-adhesive DRF at the forehead, their invasive nasopharyngeal DRF revealed improved accuracy of $25 \%$ at skull base targets. Given the endoscopic insertion and removal, the device seemed mainly targeted at otolaryngologists.

Recently, the same group described a nasopharyngeal registration tool for lateral skull base surgery in an experimental setup. ${ }^{16}$ This so-called Rhinospider was inserted into the nasopharynx prior to preoperative imaging, serv- ing as a fiducial for imaging and tracking until the end of surgery.

Catapano et al. described palatal positioning of a Medtronic round, skin-adhesive EM tracker and nonrigid fixation with gauze in 34 patients for both endoscopic endonasal and microscopic transcranial approaches. ${ }^{17}$ Error was within $3 \mathrm{~mm}$ in 33 of 34 patients (97\%).

We used a standard nasal tamponade to fix the EM sensor in the posterior part of the inferior nasal meatus. Inside the nondeformable osseous part of the nasal cavity and secured from drag on the cabling, the NT proved highly

TABLE 3. Relative deviation of the NT versus ST positions during predefined tasks

\begin{tabular}{|c|c|c|c|c|c|}
\hline \multirow[b]{3}{*}{ Task } & \multicolumn{4}{|c|}{ Deviation (mm) } & \multirow{3}{*}{$\begin{array}{c}\text { p Value } \\
\text { (paired } \\
\text { t-test) }\end{array}$} \\
\hline & \multicolumn{2}{|c|}{ ST } & \multicolumn{2}{|c|}{ NT } & \\
\hline & Median & Range & Median & Range & \\
\hline Sterile prep & 0.3 & $0.1-1.7$ & 0.1 & $0.0-0.8$ & 0.025 \\
\hline Draping & 1.3 & $0.1-3.5$ & 0.3 & $0.0-1.3$ & 0.002 \\
\hline Skin incision & 0.2 & $0.0-1.6$ & 0.1 & $0.0-2.0$ & NS \\
\hline Flap fixation & 1.6 & $0.0-7.4$ & 0.2 & $0.0-2.8$ & 0.002 \\
\hline Craniotomy & 0.3 & $0.0-2.0$ & 0.2 & $0.0-1.4$ & NS \\
\hline Microscope start to end & 0.3 & $0.0-11.2$ & 0.3 & $0.0-1.0$ & NS \\
\hline
\end{tabular}


stable throughout the procedure without invasive fixation. Insertion and removal can be easily performed without the use of an endoscope. Patient-to-image registration can be performed as usual with surface merge, skin fiducials, or bone screws.

\section{Stability of the Nasal DRF}

Continuous loss of navigation accuracy with optic tracking and a head clamp has been systematically analyzed by Stieglitz et al. ${ }^{7}$ Besides the known factors such as positioning, lesion localization, type of imaging, and mode of registration, they report a continuous decline in accuracy at draping, skin retraction, and craniotomy and with the duration of surgery. Draping caused a mean $2.7 \mathrm{~mm}$ decline in accuracy. In our present study, the ST also deviated from its original position during draping (median 1.3 $\mathrm{mm}$ ), whereas the NT remained significantly more stable. We hypothesize that the weight of the surgical drapes exerts traction on the patient's skin that affects the ST but is not transmitted into the nasal cavity.

Skin retraction with spring hooks caused a mean decline of $1.2 \mathrm{~mm}$ in accuracy in the study by Stieglitz et al. ${ }^{7}$ This was attributed to traction on the galeal flap causing relative movements between head and DRF at the level of the skull clamp. Hence, if we anticipated flap retraction by spring hooks, we fixed the RT directly to the bone rather than to the skull clamp. Further, we attached the ST at a position most unrelated to the flap elevation. Using this setup, we did not observe considerable deviation gain during flap fixation with the NT, but with the ST (median 1.6 $\mathrm{mm})$, possibly due to movement of the whole scalp even with distant skin retraction.

Craniotomy did not result in a significant decrease in accuracy in our study. Stieglitz et al. reported a decrease of $1 \mathrm{~mm}$ in accuracy during craniotomy, possibly from movements of the skull clamp.

Duration of surgery also increased error in the study by Stieglitz et al. (1.3 $\mathrm{mm}$ at 30 minutes and $4.4 \mathrm{~mm}$ at 5.5 hours)..$^{7}$ This continuous loss of accuracy was attributed to shift between head and head clamp due to continuous traction on the head by spring retractors, the patient, and drape weight. In contrast, the NT was continuously accurate throughout the procedure, possibly because of its position independent of the head clamp.

In sum, the NT was relatively unaffected by intraoperative tasks and by the duration of surgery with a median deviation of $1.3 \mathrm{~mm}$ (range $0.5-3.0 \mathrm{~mm}$ ) at the end of microsurgery, likely because of its secure position inside the nasal cavity. Given the mobility of skin, the ST was significantly more affected (median deviation $4.0 \mathrm{~mm}$, range $1.2-11.2 \mathrm{~mm}$ at the end of microsurgery, $\mathrm{p}=0.002$ ).

\section{Potential Applications of the NT}

Rigid head fixation is not required with EM tracking, as the DRF is directly fixed to the patient's head. Consequently, repositioning of the patient's head is possible at any time during surgery without the need for re-registration and without the loss of accuracy if the NT is used. The NT provides the possibility to navigate ventricular catheters with high precision in the ICU or emergency room where skull clamping is unavailable or to perform regular shunting procedures in which skull clamping is unacceptably invasive. Further, if the NT is inserted into a smaller nasal dressing, its application can be extended to pediatric (shunting) procedures, obviating the need for rigid head fixation to avoid skull fractures and epidural hematomas. ${ }^{3}$

With the NT, registration can always be performed with the patient placed supine prior to final positioning. Future studies will clarify if this can improve registration for side and prone positions, in which registration after positioning is cumbersome and less accurate. ${ }^{18}$

\section{Device Limitations}

Because of the technologically more complex EM field-detecting devices marketed as single-use products, EM navigation is considerably more expensive than optic tracking. Hence, its application should be limited to specific indications when constant tracking is important (e.g., in intrinsic brain tumor surgery ${ }^{4}$ ), when tip-tracking of catheters is required (e.g., in shunt procedures), or when pinning with a skull clamp may be too invasive (e.g., in pediatric cases or endoscopic procedures $\left.{ }^{19}\right)$. However, as the rate of EM procedures increases, the economics of scale may lead to a decrease in cost.

Because of the distortions of the EM field caused by ferromagnetic material, nonferromagnetic head holders should be used. However, standard metal skull clamps and instruments can still be applied with experience.

Because of its position inside the nasal cavity, the NT was not applicable for endoscopic transnasal skull base surgery. A specialized oral tracker is currently being developed for this purpose.

The NT assembly is not commercially available at present. However, the EM coil array can be easily harvested from its nonwaterproof housing of the wing-style AxiEM tracker. Nasal tamponades are widely available, and manual assembly of the NT did not significantly add to operating room time.

The proposed NT assembly may be too large for some nostrils, for example, in pediatric patients. In these cases, a smaller nasal tamponade can be considered or the tamponade completely omitted.

\section{Complications}

Application of a dry nasal tamponade may be fraught with nasal hemorrhage due to mucosal tears. As soaking the NT in water impeded proper application to the posterior part of the inferior meatus, we preferred to cover only the tamponade surface with antibiotic ointment prior to insertion. Minor epistaxis was encountered in only one case in our series immediately after removal of the NT and did not require any further intervention.

\section{Conclusions}

Application of the EM endonasal patient tracker was feasible and highly stable ex vivo as well as in the clinical setting. Thus, our innovation combines the advantages of high precision and noninvasiveness and may, in the future, enhance EM navigation-guided neurosurgery. 


\section{References}

1. Grunert P, Darabi K, Espinosa J, Filippi R. Computer-aided navigation in neurosurgery. Neurosurg Rev. 2003;26(2):73101.

2. Mehbodniya AH, Moghavvemi M, Narayanan V, Waran V. Frequency and causes of line of sight issues during neurosurgical procedures using optical image-guided systems. World Neurosurg. 2019;122:e449-e454.

3. Hayhurst C, Byrne P, Eldridge PR, Mallucci CL. Application of electromagnetic technology to neuronavigation: a revolution in image-guided neurosurgery. J Neurosurg. 2009;111(6):1179-1184.

4. Mert A, Gan LS, Knosp E, et al. Advanced cranial navigation. Neurosurgery. 2013;72(suppl 1):43-53.

5. Zaaroor M, Bejerano Y, Weinfeld Z, Ben-Haim S. Novel magnetic technology for intraoperative intracranial frameless navigation: in vivo and in vitro results. Neurosurgery. 2001;48(5):1100-1108.

6. Chartrain AG, Kellner CP, Fargen KM, et al. A review and comparison of three neuronavigation systems for minimally invasive intracerebral hemorrhage evacuation. J Neurointerv Surg. 2018;10(1):66-74.

7. Stieglitz LH, Fichtner J, Andres R, et al. The silent loss of neuronavigation accuracy: a systematic retrospective analysis of factors influencing the mismatch of frameless stereotactic systems in cranial neurosurgery. Neurosurgery. 2013;72(5):796-807.

8. Lin L, Gao Y, Chai G, et al. Electromagnetic navigation in craniofacial surgery based on automatic registration of dental splints. J Craniofac Surg. 2020;31(2):393-396.

9. Soteriou E, Grauvogel J, Laszig R, Grauvogel TD. Prospects and limitations of different registration modalities in electromagnetic ENT navigation. Eur Arch Otorhinolaryngol. 2016;273(11):3979-3986.

10. Rosenow JM, Sootsman WK. Application accuracy of an electromagnetic field-based image-guided navigation system. Stereotact Funct Neurosurg. 2007;85(2-3):75-81.

11. Mascott CR. Comparison of magnetic tracking and optical tracking by simultaneous use of two independent frameless stereotactic systems. Neurosurgery. 2005;57(4)(suppl):295-301.

12. Schicho K, Figl M, Donat M, et al. Stability of miniature electromagnetic tracking systems. Phys Med Biol. 2005;50(9):2089-2098.

13. Grayeli AB, Esquia-Medina G, Nguyen Y, et al. Use of anatomic or invasive markers in association with skin surface registration in image-guided surgery of the temporal bone. Acta Otolaryngol. 2009;129(4):405-410.
14. Bernardeschi D, Nguyen Y, Villepelet A, et al. Use of bone anchoring device in electromagnetic computer-assisted navigation in lateral skull base surgery. Acta Otolaryngol. 2013;133(10):1047-1052.

15. Kral F, DiFranco M, Puschban J, et al. A new nasopharyngeal dynamic reference frame improves accuracy in navigated skull base targets. Surg Innov. 2014;21(3):283-289.

16. Bárdosi Z, Plattner C, Özbek Y, et al. CIGuide: in situ augmented reality laser guidance. Int J Comput Assist Radiol Surg. 2020;15(1):49-57.

17. Catapano G, Sgulò FG, Acurio Padilla PE, et al. Palatal position of patient tracker for magnetic neuronavigation system: technical note. World Neurosurg. 2018;116:105-109.

18. Ogiwara T, Goto T, Aoyama T, et al. Bony surface registration of navigation system in the lateral or prone position: technical note. Acta Neurochir (Wien). 2015;157(11):20172022.

19. Martínez-Moreno M, Widhalm G, Mert A, et al. A novel protocol of continuous navigation guidance for endoscopic third ventriculostomy. Neurosurgery. 2014;10(suppl 4):514-524.

\section{Disclosures}

Dr. Wolfsberger is currently an educational consultant for Medtronic Surgical Technologies and, with Medtronic Navigation, has filed a US patent application.

\section{Author Contributions}

Conception and design: Wolfsberger, Cervera-Martinez. Acquisition of data: Wolfsberger, Wurzer, Cervera-Martinez, Micko, Kronreif. Analysis and interpretation of data: Wolfsberger, Wurzer, Minchev, Micko, Kronreif. Drafting the article: Wurzer, Cervera-Martinez. Critically revising the article: Wurzer, Minchev, Micko. Approved the final version of the manuscript on behalf of all authors: Wolfsberger. Statistical analysis: Wolfsberger, Wurzer. Administrative/technical/material support: Minchev, Micko, Kronreif. Study supervision: Wolfsberger.

\section{Correspondence}

Stefan Wolfsberger: Medical University Vienna, Austria. stefan. wolfsberger@meduniwien.ac.at. 